\title{
Ankle Arthroscopy, Lateral Ligament Repair and Peroneal Tendon Reefing for Chronic Lateral Ankle Instability: The Triad vs Arthroscopy with Ligament Repair
}

\author{
John J. Anderson, Loren K. Spencer, Zflan Fowler \\ Alamogordo Orthopedics, Alamogordo, NM, USA \\ Email: josephanderson5@gmail.com
}

Received 18 July 2015; accepted 5 September 2015; published 8 September 2015

Copyright (C) 2015 by authors and Scientific Research Publishing Inc.

This work is licensed under the Creative Commons Attribution International License (CC BY). http://creativecommons.org/licenses/by/4.0/

(c) (;) Open Access

\begin{abstract}
Peroneal tendon and retinacular pathology contributes to chronic lateral ankle instability. The "gold standard" surgical treatment for chronic lateral ankle instability has been the BroströmGould procedure with its modifications. An ankle arthroscopy is an adjuvant procedure to address any intra-articular pathology. We review our results of two study groups. The first group underwent an ankle arthroscopy and a Broström-Gould procedure. The second group (triad) underwent an ankle arthroscopy, the Broström-Gould procedure and excision of low lying peroneal muscle belly with tightening of the inferior peroneal retinaculum. The triad technique was performed on 97 patients and results compared to 71 ankle arthroscopies with the Broström-Gould procedure. The patients were contacted at a mean follow-up time of 30 months for postoperative ACFAS scores, VAS scores, and overall satisfaction. Patients were also asked to report incidence of ankle sprain recurrence. The average postoperative ACFAS hindfoot and ankle score was 92 for the triad group and 89 for the arthroscopic debridement and Broström-Gould group. The average overall satisfaction was $98 \%$ in the triad group and $91 \%$ in the scope and Broström group. There were 4 $(4.3 \%)$ recurrences in the triad group and $12(17.6 \%)$ in the Broström-Gould with scope group. Each group had a similar incidence of wound healing complications. Peroneal tendon and retinacular pathology contributes to continued ankle instability and pain. We believe that the triad procedure is superior to the Broström-Gould procedure.
\end{abstract}

\section{Keywords}

Broström-Gould, Lateral Ankle Instability, Triad Procedure 


\section{Introduction}

Chronic lateral ankle instability is a documented complication following $10 \%$ to $30 \%$ of significant ankle sprains [1] [2]. Some reports suggest that chronic instability remains about the lateral ankle as frequently as $40 \%$ of the time leading to high re-injury rates with increased and repetitive use of the same unstable ankle [2]. Many surgical procedures have been described to reconstruct the lateral ankle ligament complex [3]. Commonly, the peroneal tendons are used to perform these repairs in either a direct, indirect, anatomic manner or a non-anatomic manner. The Broström procedure, with or without the Gould modification, is considered by many foot and ankle surgeons to be the "gold standard" for persistent lateral ankle instability or recurrent later ankle sprains [3]. Many surgeons augment their modified Broström procedures with ankle arthroscopy to assist in evaluation of any intra-articular pathology and also reduce any intra-articular synovitis [4] [5].

Lateral peroneal pain is commonly associated with chronic ankle instability [5]. It is the author's belief that a low lying peroneus brevis muscle belly can lead to attenuation of the inferior portion of the peroneal retinaculum resulting in painful symptoms following a severe ankle inversion injury [6]. It is the authors' hypothesis that peroneal pathology contributes to lateral ankle instability, specifically a low lying muscle belly, synovitis of the tendons themselves or these in combination with a laxity of the distal peroneal retinaculum. Chronic synovitis, longitudinal rents or tears in the peroneal brevis, and incomplete or complete tears in the peroneal retinaculum can also lead to an inability of the peroneal tendons to react in a normal proprioceptive manner. Neither intraarticular repair of ankle ligaments nor the Broström-Gould procedure with its modifications addresses peroneal pathology [7].

The purpose of this study was to compare the postoperative course, objective and subjective improvement between two study groups. The first group underwent an ankle arthroscopy and the standard Broström-Gould procedure. The second group (triad) underwent an ankle arthroscopy, the Broström-Gould procedure and excision of low lying peroneal muscle belly and/or tightening of the inferior peroneal retinaculum, including a tenosynovectomy as indicated intraoperatively. The board of ethics at the local hospital reviewed the cases and granted permission for the procedures and resulting research.

\section{Patients and Methods}

A consecutive series of 993 patients were treated between August 2003 and May 2008 for acute ankle sprains in the primary author's clinic. All patients were initially treated conservatively with immobilization and/or ankle bracing and/or physical therapy. Patients who failed conservative treatment were considered for surgical treatment. Those patients who, by informed consent, elected for surgery reported unresolved anterior lateral ankle gutter margin pain. All procedures were performed by the same surgeon (J.J.A.) and were done at two outpatient surgery centers and one outpatient hospital. An American College of Foot and Ankle Surgeons (ACFAS) hind foot ankle score system was used to obtain preoperative subjective scores [8]. All patients were interviewed by the author and staff to collect data for the ACFAS and visual analog scale (VAS) scores. Patients were examined by the primary author and the criteria for performing the triad procedure was retrofibular pain or swelling with or without concurrent MRI findings. A subgroup labeled "worker's compensation" was also evaluated that included 22 work injuries in which payment was typical. All data collected and statistically analyzed using student's t-test or ANOVA.

Patients were excluded from the study due to diagnosis of fracture, osteochondral defect, previous surgery, inadequate fibular groove, or anterior lateral ankle impingement. Also excluded were patients with peroneal tendon pathology, including peroneal subluxation, requiring direct repair, as peroneal tendon dysfunction was most likely the primary etiology rather than standard instability. Patients with neurologic or connective tissue disease were also excluded from the study.

Of 993 patients, 168 patients required surgical intervention. There were 71 patients in the Broström-Gould group (scope with lateral ligament repair) and 97 patients in the triad group (ankle arthroscope, ligament repair, and peroneal tendon reefing). Of these patients, a total of 68 in the Broström-Gould group and 92 in the triad group were available for follow up. The patients were divided between the two groups based on non-resolving peroneal tendon pain. The Broström-Gould patients had a lack of pain in the retrofibular region with or without MRI findings of peroneal tendonitis or LLMB. Those selected for the triad group had pain in the peroneal region during the clinical exam. A lax peroneal reticulum with pain generated in the retrofibular peroneal pouch on anterior drawer testing and MRI findings of low-lying muscle belly with accompanying synovitis and flattening of 
the peroneus brevis.

The patients underwent surgery at an average of 6 months post injury with a range of 2.5 to 38 months. Follow up appointments were scheduled at 2 week intervals for the first 3 months after which additional appointments were made based on individual patient progress. The patients were contacted at a mean follow up of 30 months (ranged 6 to 90 mo.) for postoperative ACFAS scores, VAS scores and overall satisfaction (Yes or No). Patients were also asked to report the number of ankle sprains since surgery. A single sprain was considered a re-injury; whereas, multiple sprains with continued instability clinically, subjectively and objectively by stress radiograph were considered recurrent. Retrospective chart review was performed in order to determine the number and type of complications as well as individual patient postoperative course. The preoperative ACFAS scores were tabulated after all other data had been obtained.

\subsection{Surgical Technique and Post-Operative Management: Broström-Gould Procedure}

A thigh tourniquet was applied and the standard medial and lateral ankle portals were entered with assistance from an ankle distracting unit. Gravity pressure was used to maintain joint distension. A full diagnostic arthroscopy including portal exchange occurred. Direct visualization of gross instability with forced inversion and anterior drawer was assessed. Arthroscopic ankle joint debridement of all scar and synovitic tissue was performed. Periosteum from the intra-articular aspect of the fibula was also resected. Pre and post debridement photos were taken and the scopes removed. The medial portal was closed using 4-0 nylon.

The lateral portal was extended to the distal tip of the fibula. Standard care was taken to avoid contact with neurovascular structures. Non-absorbable \#0, \#1 and \#2-0 vicryl were used to re-approximate the anatomic alignment of the anterior talofibular and calcaneofibular ligaments and perform a vest-over-pants reefing of the lateral aspect of the extensor retinaculum or the Gould modification. The remaining incisions were closed utilizing 4-0 nylon. A modified cast splint was applied with the foot held in a dorsiflexed and everted position. The patient transitioned to an equalizer boot with passive range of motion at 2 weeks. Full weight bearing and physical therapy commenced at 4 to 8 weeks. The patient was allowed to return to full activity and sports at $8-12$ weeks with an ankle stirrup brace.

\subsection{Surgical Technique Post-Operative Management: Triad Procedure}

The triad group consisted of a medial and lateral standard arthroscopy with portal exchange. Any intraarticular pathology was addressed similar to the Broström-Gould group. Following the ankle scope, a modified curvilinear incision was made directly over the lateral ankle gutter (Figures 1-6). Dissection was carried through the subcutaneous tissue exposing and incising the peroneal retinaculum. If present, low lying peroneal muscle belly was debrided to approximately $5 \mathrm{~cm}$ proximal to the distal tip of the fibula. If synovitic or mucinous changes were present in the peroneal tendons, an open synovectomy was performed. The peroneal retinaculum was repaired in vest-over-pants type fashion along with the standard Broström-Gould modification utilizing \#0, \#1,

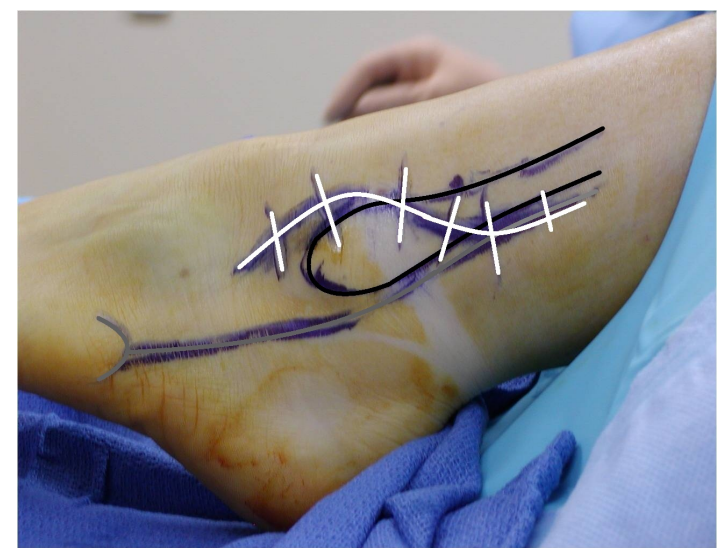

Figure 1. Incision placement triad procedure: White: planned incision; Black: fibula; Grey: peroneal tendons with 5th Metbase. 


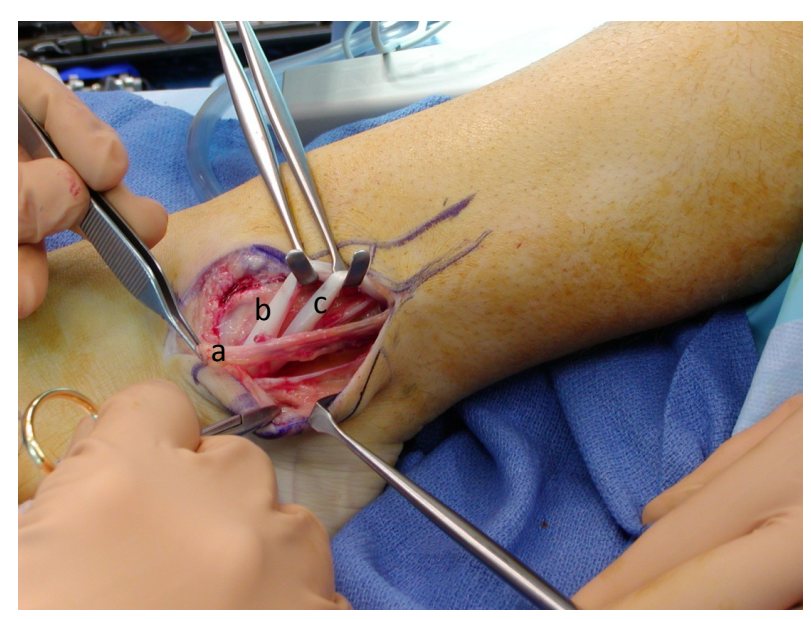

Figure 2. (a) Peroneal retinaculum, (b) brevis and (c) longus after synovectomy.

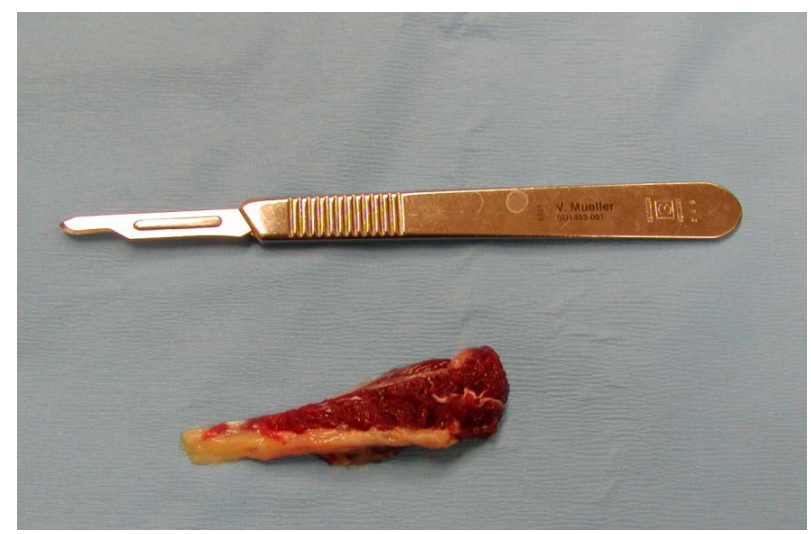

Figure 3. Excision of low lying muscle belly of peroneus brevis.

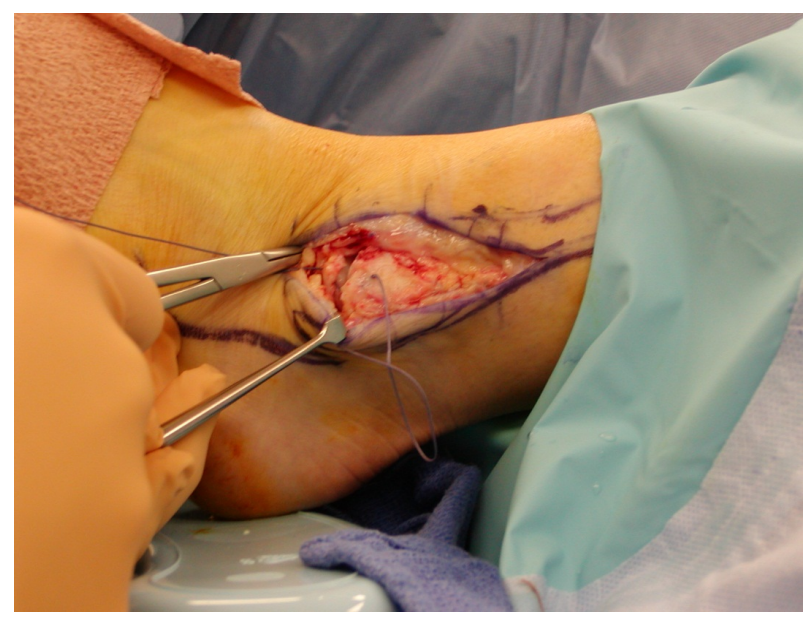

Figure 4. After retinacular ATF and CF ligament repair.

and \#2-0 vicryl and 4-0 nylon for skin closure. The triad group followed the same postoperative course as the Broström-Gould and scope group. A modified cast splint was applied for 2 weeks following transition to full weight bearing and return to full activity and sports at 8 - 12 weeks with an ankle stirrup brace. 


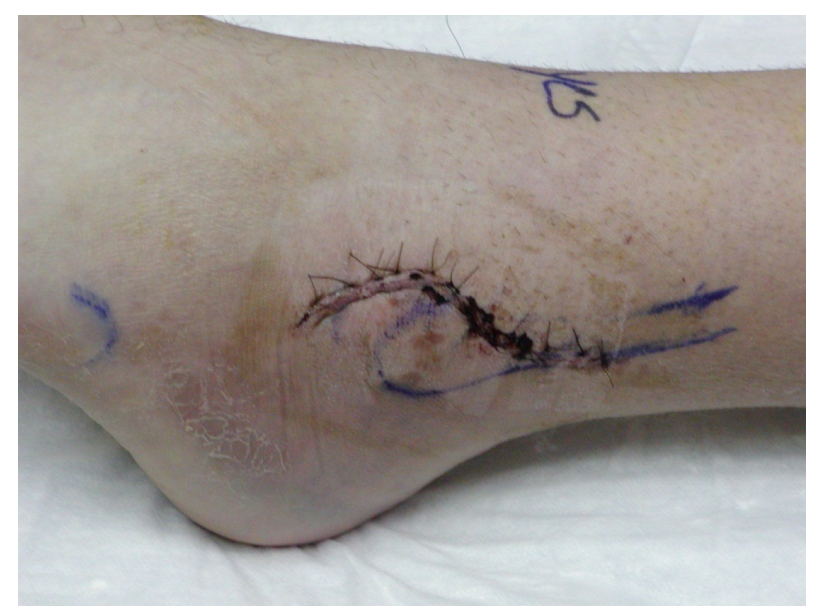

Figure 5. 2 week postop follow-up.

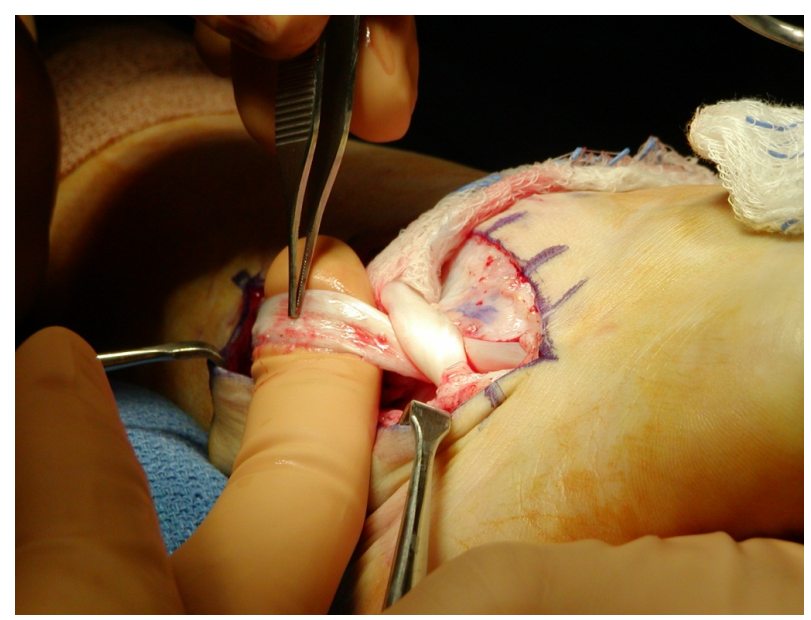

Figure 6. Tenosynovitis of peroneus brevis.

\section{Results}

Of 993 patients seen for acute ankle sprains, a total of 724 responded to conservative care. 269 (27.1\%) returned with chronic ankle instability after failing standard treatment including outpatient physical therapy and immobilization. Of the 269 chronic ankle instability patients, 30 (3\%) patients elected to continue with an ankle support brace indefinitely and not proceed with surgery. 168 (16.9\%) went on to surgery, 71 underwent modified Broström-Gould and ankle arthroscopy and 97 underwent the triad procedure of which 68 and 92 patients were available for follow up, respectively.

There were 95 females and 73 males. The mean age was 39.3 with a range from 14 to 81 years. The average age for females and males in our study was 42.3 and 36.7, respectively. This supports average ages seen in previous studies by Karlsson et al. [9]. Using chi-squared analysis, the two procedure groups were compared and it was determined there was no statistically significant difference between them (Table 1).

Preoperative MRI demonstrated a peroneal muscle belly extending at or past the distal tip of the fibula in 156 ( 92\%) of the total patients: 70 of the 71 patients in the Broström and scope group and 86 of the 97 patients in the triad group. This diagnosis was confirmed intra-operatively. A total of 45 patients exhibited tenosynovitis of the peroneus brevis, peroneus longus or both on MRI. A total of 12 patients had confirmed intrasubstance or longitudinal tears or rents of the peroneus brevis tendon. An additional 29 patients had confirmed intra-articular loose bodies, 14 of which patients had confirmed osteochondral lesions of the talus.

71 (7.2\%) patients were excluded from the study. 38 patients had significant peroneal tendon dysfunction, including complete longitudinal tears of either or both peroneal tendons or peroneal subluxation which required 
Table 1. Demographics.

\begin{tabular}{ccccccc}
\hline & $\begin{array}{c}\text { Total \# } \\
\text { (Actual) }\end{array}$ & Age (avg) & Smoker & $\begin{array}{c}\text { Male/ } \\
\text { Female }\end{array}$ & Comorbidities $^{\mathbf{b}}$ & $\begin{array}{c}\text { Worker's } \\
\text { Comp }\end{array}$ \\
\hline $\begin{array}{c}\text { Broström } \\
+ \text { Scope }\end{array}$ & $\begin{array}{c}71 \\
(68)\end{array}$ & 41.1 & $17(24 \%)$ & $31 / 40$ & $11(15.5 \%)$ & $9(12.7 \%)$ \\
Triad & 97 & 37.9 & $15(15.5 \%)$ & $42 / 55$ & $13(13.4 \%)$ & $13(13.4 \%)$ \\
P-value & $(92)$ & 0.260 & 0.167 & 0.963 & 0.574 & 0.462 \\
\hline
\end{tabular}

Total patients with ankle instability 993: 71 (7\%) excluded, 754 (76\%) treated conservatively, and 168 (17\%) treated surgically. a: Actual number excludes patients lost to follow-up; b: Broström + Scope-6 obese, 4 diabetes, 1 rheumatoid arthritis; Triad-6 obese, 7 diabetes.

extensive repair, retrofibular groove deepening, and/or peroneal tenodesis. An additional 12 patients with significant osteochondral lesions (greater than $0.5 \mathrm{~cm}^{2}$ ) were excluded given that significant joint pain was believed to be the primary cause for their continued pain. 11 patients which required recurrent or revisional surgery with a modification of a peroneal tendon transfer or lateral ankle ligament reconstruction were excluded. Also excluded were 5 patients with significant anterior-lateral osteophytosis of the tibia and/or fibula causing lateral pain, impingement, and instability which warranted an open exostectomy. Finally, 2 patients with mild cerebral palsy and 3 patients with underlying connective tissue disorders were excluded.

Patients from each group were contacted and evaluated using a ACFAS hindfoot and ankle scoring scale to obtain a final end point score. The average preoperative ACFAS score was 76 in the Broström-Gould with ankle arthroscopy group and 75 in the triad group with a range between 66 and 90. Following surgery, the average ACFAS score was 89 in the Broström-Gould with ankle arthroscopy group and 92 in the triad group. The percentage of patients satisfied was $91 \%$ in the Scope and Broström group and $98 \%$ in the triad group. This approached significance with a p-value of 0.05 . The average VAS satisfaction score was 4.1 for the BroströmGould group and 4.6 for the triad group. There was no statistically significant difference between preoperative, postoperative and VAS scoring between the procedure groups (Table 2).

In the work injury group, the average preoperative ACFAS score was 69 with the Broström-Gould and ankle arthroscopy procedure and 70 with the triad procedure. Postoperative ACFAS scores were 81 and 85, respectively. The percentage of work injury patients satisfied was $77 \%$ in the Scope and Broström group and $85 \%$ in the triad group. The average VAS satisfaction score was 3.6 for the Broström-Gould group and 3.7 for the triad group. Postoperative satisfaction and VAS scores between the worker's compensation and all patients approached significance with p-values of 0.067 and 0.056 , respectively (Table 3).

Of the Broström-Gould group 34 (50\%) of the patients had another ankle sprain and 12 (17.6\%) of all those patients had continued chronic ankle instability. In the triad group only $10(10.9 \%)$ of the patients had a reinjury and 4 (4.3\%) had continued recurrent instability with multiple sprains. The triad group was statistically less likely to have a re-injury $(\mathrm{p}<0.001)$ or recurrence $(\mathrm{p}=0.006)$ when compared to the ankle scope and Broström group. Complications were minor and infrequent in both groups. Three patients in the Broström-Gould group and 4 patients in the triad group had non-persistent numbness over the superficial peroneal nerve laterally. No patient had deep venous thrombosis. Six patients in the triad group and 3 patients in the Broström-Gould group had superficial dehiscence of the distal aspect of the incision which healed with superficial wound care. Other complaints noted in each group were direct pain in the lateral ankle gutter margin which resolved spontaneously with long term follow up. Patients in both groups also reported postoperative intermittent edema for the first 6 months. "Clicking without pain" was also noted in 8 patients in the triad group and no patients in the Broström and scope group. It was not noted if this was present preoperatively in these patients (Table 4).

Patients in the triad group spent statistically more time in an ankle brace than did patients in the ankle scope with Broström group $(\mathrm{p}=0.007)$. They averaged 7.25 weeks in an ankle brace compared to 6.28 weeks in Broström and ankle scope group. These patients also took significantly longer to return back to normal activity, 11.7 weeks, instead of the average 10.4 weeks reported in the Broström and ankle scope group $(\mathrm{p}=0.001)$ (Table 5). 
Table 2. Modified ACFAS/satisfaction and VAS scores.

\begin{tabular}{ccccccc}
\hline & \multicolumn{3}{c}{ All Patients (A) } & \multicolumn{3}{c}{ Worker's Comp Only (B) } \\
& Preop & Postop & \# Satisfied (VAS) & Preop & Postop & \# Satisfied (VAS) $^{\mathbf{a}}$ \\
\hline Broström + Scope & 76 & 89 & $61(91 \%)(4.1)$ & 69 & 81 & $7(77 \%)(3.6)$ \\
Triad & 75 & 92 & $90(98 \%)(4.6)$ & 70 & 85 & $11(85 \%)(3.7)$ \\
Avg & 75.3 & 90.6 & $151(94.4 \%)(4.4)$ & 69.6 & 83.3 & $18(82 \%)(3.66)$ \\
p-value & 0.483 & 0.351 & $0.05(0.20)$ & 0.338 & 0.373 & $0.466(0.350)$ \\
\hline
\end{tabular}

a: 5 point Visual Analog Score.

Table 3. P values: ACFAS and VAS scores.

\begin{tabular}{cccc}
\hline & Preop & Postop & VASa Work comp. (all patients) \\
\hline Broström + Scope & 0.481 & 0.440 & $0.238(0.396)$ \\
Triad & 0.481 & 0.376 & $0.065(0.059)$ \\
Avg & 0.366 & 0.212 & $0.067(0.056)$ \\
\hline
\end{tabular}

a: 5 point Visual Analog Score.

Table 4. Complications.

\begin{tabular}{cccccc}
\hline & Numbness & Persistent Edema & Dehiscence & Re-injury $^{\mathrm{a}}$ & Recurrence $^{\mathrm{b}}$ \\
\hline Broström + Scope & $3(4.4 \%)$ & $13(19.1 \%)$ & $3(4.4 \%)$ & $34(50 \%)$ & $12(17.6 \%)$ \\
Triad & $4(4.3 \%)$ & $13(14.1 \%)$ & $6(6.5 \%)$ & $10(10.9 \%)$ & $4(4.3 \%)$ \\
p-value & 0.359 & 0.265 & 0.411 & $<0.001$ & 0.006 \\
\hline
\end{tabular}

a: Single postoperative sprain; b: Multiple postoperative sprains.

Table 5. Postoperative course.

\begin{tabular}{cccc} 
& Splint/Boot (weeks) & Ankle Brace (weeks) & Return to Activity (weeks) \\
\hline Broström + scope & 4.13 & 6.28 & 10.4 \\
Triad & 3.92 & 7.25 & 11.7 \\
p-value & 0.097 & 0.007 & 0.001 \\
\hline
\end{tabular}

\section{Discussion}

Surgical treatment of lateral ankle instability has been described extensively for repair of the ankle ligament complex following acute injury. The Broström procedure with the Gould modification, consisting of direct and anatomic repair of the anterior-talofibular and the calcaneofibular ligaments, is certainly less invasive and easier to perform than many of the other described procedures [10]. Ankle arthroscopy has been advocated in the recent decade as being an excellent adjunct to addressing intra-articular pathology such as anterior lateral ankle impingement [10]. Repair of long-term instability can be combined with anatomic or non-anatomic transfer of tendon, tensor fascia lata, and/or other grafts [11].

It is the authors' belief that peroneal tenosynovitis and the presence of a low lying peroneal muscle belly should be addressed if concurrent with chronic instability. The MRI findings of the study demonstrated a higher prevalence of a low-lying peroneal muscle belly than previous reports: $92 \%$ of the patients had a low-lying pe- 
roneal muscle belly compared to previous, older reports of 30\% in the normal population [12]. This discrepancy may be due to patients with recurring ankle sprains and instability may have a higher incidence of LLMB than the normal population. A lax peroneal retinaculum or extensive synovitis may have a lower proprioceptive response or perhaps even less on the ability for the peroneals to stabilize the lateral ankle. This study would suggest that long-term excellent results can be achieved by both an ankle arthroscopy with an open Broström-Gould modification and a tightening or reefing of the inferior portion of the peroneal retinaculum with excision of a low lying peroneal muscle belly. The authors maintain that the inferior portion of the peroneal retinaculum is significant to the functionality of the peroneus brevis and peroneus longus tendons which are a major stabilizing force for the lateral ankle complex. The authors feel that the ankle ligaments provide only a portion of the overall stability of the ankle joint while the peroneal tendons form the lateral harness and sling to the ankle.

The authors recognize the multifactorial etiologies associated with lateral ankle instability and feel that, in combination with the open modified Broström Gould, ankle arthroscopy enables one to address more contributing intra-articular pathologies. The results of the study demonstrate that peroneal tendon reefing improves the stability of the lateral ankle complex by stabilizing the forces of the peroneus brevis and the peroneus longus tendons. The authors advocate first line treatment with immobilization and physical therapy for all significant ankle sprains. If conservative treatment fails and physical exam and MRI corroboration demonstrate tenderness or swelling over the peroneal tendons or within the retrofibular complex, the threshold should be lowered for cleaning and reefing of the peroneal tendons and retinaculum.

Although recovery time from the Triad procedure was on average longer than the standard Broström and ankle scope procedure, the patients reported higher satisfaction scores, ACFAS scores and fewer recurrent injuries. The addition of excising the low lying peroneal muscle belly with tightening of the inferior peroneal retinaculum in addition to the Broström-Gould procedure and ankle arthroscopy showed overall improvement post-surgery and stability of the ankle. The authors recognize the complexity involved with treatment of chronic ankle instability and also the limitations of this study, such as the lack of long-term follow-up of patients (average 30 months), not separating the athletic patients from the low demand patients and the possibility of the worker's compensation group skewing the results due to the individuals receiving financial reimbursement for the time of their recovery, which may influence them to take more time than necessary [13]. Ankle arthroscopy, lateral ligament repair and peroneal tendon reefing techniques, in combination, should be included in the arsenal for treatment of chronic lateral ankle instability.

\section{References}

[1] Karlsson, J. and Lansinger, O. (1992) Lateral Instability of the Ankle Joint. Clinical Orthopaedics, 276, $253-261$. http://dx.doi.org/10.1097/00003086-199203000-00035

[2] Ferkel, R.D. and Chams, R.N. (2007) Chronic Lateral Instability: Arthroscopic Findings and Long Term Results. Foot \& Ankle International, 28, 24-31. http://dx.doi.org/10.3113/FAI.2007.0005

[3] Baumhauer, J.F. and O’Brien, T. (2002) Surgical Considerations in the Treatment of Ankle Instability. Journal of Athletic Training, 37, 458-462.

[4] Hua, Y., Chen, S. and Li, Y. (2010) Combination of Modified Brostrom Procedure with Ankle Arthroscopy for Chronic Ankle Instability Accompanied by Intra-Articular Symptoms. Arthroscopy, 26, 524-528. http://dx.doi.org/10.1016/j.arthro.2010.02.002

[5] Oloff, L.M., Bocko, A.P. and Fenton, G. (2000) Arthroscopic Monopolar Radiofrequency Thermal Stabilization for Chronic Lateral Ankle Instability: A Preliminary Report on 10 Cases. Journal of Foot and Ankle Surgery, 39, 144-153. http://dx.doi.org/10.1016/S1067-2516(00)80015-0

[6] Freccero, D.M. and Berkowitz, M.J. (2006) The Relationship between Tears of the Peroneus brevis Tendon and the Distal Extent of Its Muscle Belly: An MRI Study. Foot \& Ankle International, 27, 236-239.

[7] Heckman, D.S., Reddy, S., Pedowitz, D., Wapner, K.L. and Parekh, S.G. (2008) Current Concepts Review: Operative Treatment for Peroneal Tendon Disorders. Journal of Bone \& Joint Surgery, 90-A, 404-418. http://dx.doi.org/10.2106/JBJS.G.00965

[8] Thomas, J.L., Christensen, J.C., Mendicino, R.W., Schuberth, J.M., Weil, L.S. and Zlotoff, H.J. (2005) ACFAS Scoring Scale User Guide. Journal of Foot and Ankle Surgery, 44, 316-335. http://dx.doi.org/10.1053/j.jfas.2005.07.012

[9] Karlsson, J. and Lansinger, O. (1992) Lateral Instability of the Ankle Joint. Clinical Orthopaedics, 276, 253-261. http://dx.doi.org/10.1097/00003086-199203000-00035

[10] Keller, M. and Grossman, J. (1996) Lateral Ankle Instability and the Brostrom-Gould Procedure. Journal of Foot and 
Ankle Surgery, 35, 513-520. http://dx.doi.org/10.1016/S1067-2516(96)80123-2

[11] Colville, M.R. (1994) Reconstruction of the Lateral Ankle Ligaments. Journal of Bone \& Joint Surgery, 76-A, 10921102.

[12] Domblek, M.F., Lamm, B.M., Saltrick, K., Mendicino, R.W. and Catanzariti, A.R. (2003) Peroneal Tendon Tears: A Retrospective Review. Journal of Foot and Ankle Surgery, 42, 250-258. http://dx.doi.org/10.1016/S1067-2516(03)00314-4

[13] Froom, P., Melamed, S., Nativ, T., Gofer, D. and Froom, J. (2001) Low Job Satisfaction Predicts Delayed Return to Work After Laparoscopic Cholecystectomy. Journal of Occupational and Environmental Medicine, 43, 657-662. http://dx.doi.org/10.1097/00043764-200107000-00014 